\title{
Two-dimensional arbitrarily shaped acoustic cloaks composed of homogeneous parts
}

\author{
Qi Li and Jeffrey S. Vipperman* \\ Department of Mechanical Engineering \& Materials Science, University of Pittsburgh, \\ Pittsburgh, PA 15260, USA
}

\begin{abstract}
Acoustic cloaking is an important application of acoustic metamaterials. Although the topic has received much attention, there are a number of areas where contributions are needed. In this paper, a design method for producing acoustic cloaks with arbitrary shapes that are composed of homogeneous parts is presented. The cloak is divided into sections, each of which in turn is further divided into two parts, followed by the application of transformation acoustics to derive the required properties for cloaking. With the proposed mapping relations, the properties of each part of the cloak are anisotropic but homogeneous, which can be realized using two alternating layers of homogeneous and isotropic materials. A hexagonal and an irregular cloak are presented as design examples. The full wave simulations using COMSOL Multiphysics finite element software show that the cloaks function well at reducing reflections and shadows. The variation of the cloak properties is investigated as a function of three important geometric parameters used in the transformations. A balance can be found between cloaking performance and material properties that are physically realizable.
\end{abstract}

\section{Introduction}

Acoustic metamaterials were introduced in recent years, followed by considerable focus by many researchers. A metamaterial is an engineered artificial material within which the propagation of acoustic waves can be altered. An acoustic cloak is one kind of acoustic metamaterial that can make an object acoustically transparent by bending the waves around the object.

Acoustic cloaks are analogous to electromagnetic (EM) cloaks. Pendry, et $\mathrm{al}^{1}$ first derived the properties required for EM cloaking based on transformation optics. Later, Cummer, et $\mathrm{al}^{2}$ presented 2D acoustic cloaks by showing they are analogous to EM cloaks. Later, both Cummer, et $\mathrm{al}^{3}$ and Chen and $\mathrm{Chan}^{4}$ independently extended the acoustic cloaking theory to 3D.

The extreme properties required for ideal cloaks make them difficult to fabricate. Approximate cloaks $^{5}$, formed by transforming from one cylindrical shell to another, have much improved manufacturability. The 'reduced' cloak ${ }^{6}$ and the 'ground cloak ${ }^{7}$ are a few practical examples where some cloaking ability was sacrificed, yet have been fabricated and tested. Popa, et $\mathrm{al}^{8}$ designed and experimentally demonstrated a 2D ground cloak, which, as the name suggests, can prevent scattering from objects against a reflecting plane. Zigoneanu, et $\mathrm{al}^{9}$ fabricated a 3D ground cloak with a pyramidal shape. Zhang, et $\mathrm{al}^{10}$ fabricated a 2D annular cloak based on a transmission line approach. Acoustic cloaks with more complicated geometries have not been fabricated yet.

Lai, et al ${ }^{11}$ proposed another kind of EM cloak which can cancel the effect of an object that is within a certain distance outside the cloak based on the concept of complementary media with double negative properties. Zhu, et $\mathrm{al}^{12}$ proposed a kind of acoustic cloak consisting of complementary media with single-negative properties. $\mathrm{Xu}$, et al ${ }^{13}$ proposed 
an acoustic sensor composed of a pair of homogeneous isotropic single-negative media, which has no impact on the exterior acoustic field. Kan, et al ${ }^{14}$ designed "illusion cloaks" comprised of positive-index anisotropic materials whose material parameters are non-singular and homogeneous.

The above acoustic cloaks are examples of "inertial cloaks" with anisotropic density and isotropic bulk modulus. Norris ${ }^{15}$ analyzed acoustic cloaks and found that besides inertial cloaks, pentamode materials can also be used for acoustic cloaking. Scandrett, et $\mathrm{l}^{16}$ proposed an optimization of a pentamode-layered spherical acoustic cloak. Gokhale, et $\mathrm{al}^{17}$ proposed special transformations for pentamode acoustic cloaks. Chen, et al ${ }^{18}$ proposed a $2 \mathrm{D}$ acoustic cloak assembled by pentamode lattices.

Novel recent concepts applied to cloaking include parity-time symmetric media that permits the design of an acoustic cloak that works unidirectionally. ${ }^{19}$ Peng, et $\mathrm{al}^{20}$ analyzed anomalous Floquet topological insulators for sound, whereby pressure waves can propagate along specifically designated paths.

In theory, cloaks with more complicated boundaries have been studied. Several EM cloaks of more complicated shapes have been presented based on transformation optics. ${ }^{21-24}$ Paul, et al $^{25}$ constructed regular polygonal EM cloaks with layered structures. The authors ${ }^{26}$ proposed a method for designing acoustic cloaks of arbitrary shapes. However, the properties are anisotropic and inhomogeneous. Popa and Cummer $^{27}$ presented a method of designing EM cloaks with arbitrary shapes characterized by material parameters of same-level sets. They ${ }^{28}$ further optimized the material properties of each layer. Transformation relations are not unique. Luo, et $\mathrm{al}^{29}$ used a square root function as the coordinate transformation, while Rajput and Srivastava ${ }^{30}$ used a quadratic transformation. Cummer, et $\mathrm{al}^{31}$ presented a fraction power function as the coordinate transformation, which reduced difficulty. W. Li, et $\mathrm{al}^{32}$ proposed a two dimensional near-perfect EM cloak consisting of homogeneous parts. Wang, et $\mathrm{al}^{33}$ extended it to a three-dimensional model and derived octahedral EM cloaks. T. Li, et $\mathrm{al}^{34}$ also developed acoustic cloaks with homogeneous parts in 2D and 3D. Zhu, et $\mathrm{al}^{35}$ designed a triangular cloak composed of homogeneous parts.

The above design methods are specific to the particular cloak geometry for which they are designed. A more general method for designing acoustic cloaks with arbitrary shapes composed of homogeneous parts is needed.

In this paper, a method for designing acoustic cloaks with an arbitrary shape is presented. The acoustic cloak is first divided into sections, each of which is in turn divided into two parts. Within each part of the section, transformation acoustics is used to derive the required properties of the cloak. With specific mapping relations, the derived properties are homogeneous for each part of the cloak. Two models are designed and simulated with COMSOL Multiphysics finite element software to illustrate the method. Lastly, variation of properties versus three key geometrical parameters is investigated.

\section{Methods}

Most acoustic cloaks with arbitrary shapes, as shown in FIG. 1, can be divided into sections by radial lines starting from a point within the inner boundary. The total number of sections will determine the angle between two adjacent lines (e.g. 10 sections would each have an angle of 360/10 = 36 degrees). However, there is no requirement that each section subtend the same angle. For each section, for example OCD in FIG. 1, transformation acoustics can be used to derive the properties for cloaking. The inner and outer boundaries within each section are approximated with straight lines (e.g. BE and CD in section OCD). The angle $\theta$ of the section can be varied to affect the section size and the properties of the section, as will be discussed later. For simplicity, a local coordinate system is defined with $\mathrm{O}$ as the origin and OC as the $x$ axis.

The cloak occupies the physical space, and the virtual space is an imaginary space filled with 
background medium. A two-step transformation is performed from virtual space ACDF (FIG. 2a) to physical space BCDE (FIG. 2c). In the first step, OAD (FIG. 2a) is expanded to OBD (FIG. 2b) and ACD (FIG. 2a) is compressed to BCD (FIG. 2b) with a linear transform along $x$ direction. In the second step, the FBD (FIG. 2b) obtained in the first step is compressed to EBD (FIG. 2c) with a linear transform along the OD direction. After the two steps, points in the physical space BCDE are mapped to those in the virtual space ACDF. The area OBE corresponds to the area OAF. Particularly, when $|\mathrm{OF}|$ is zero, the area OBE corresponds to the short line OA. Smaller lengths for $|\mathrm{OA}|$ and $|\mathrm{OF}|$ correspond to better cloaking performance.

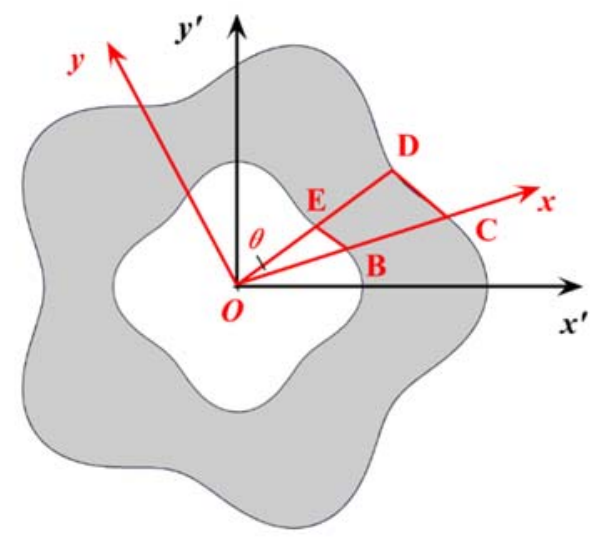

FIG. 1 A divided section and local coordinate system in an acoustic cloak with arbitrary shape.

In the first step, the points in ACD of FIG. 2a are mapped to the points in BCD of FIG. 2b using

$$
\left\{\begin{array}{l}
x_{I}=c_{1} x+c_{2} y+c_{3} \\
y_{I}=y
\end{array}\right.
$$

where,

$$
\begin{aligned}
& c_{1}=\frac{x_{C}-x_{B}}{x_{C}-x_{A}} \\
& c_{2}=\frac{x_{D}-x_{B}}{y_{D}}-\frac{x_{C}-x_{B}}{x_{C}-x_{A}} \frac{x_{D}-x_{A}}{y_{D}} \\
& c_{3}=x_{B}-\frac{x_{C}-x_{B}}{x_{C}-x_{A}} x_{A} .
\end{aligned}
$$

The points in OAD of FIG. 2a are mapped to the points in OBD of FIG. 2b using

$$
\left\{\begin{array}{l}
x_{I}=c_{4} x+c_{5} y \\
y_{I}=y
\end{array}\right.
$$

where,

$$
\begin{aligned}
& c_{4}=\frac{x_{B}}{x_{A}} \\
& c_{5}=\left(1-\frac{x_{B}}{x_{A}}\right) \frac{x_{D}}{y_{D}} .
\end{aligned}
$$
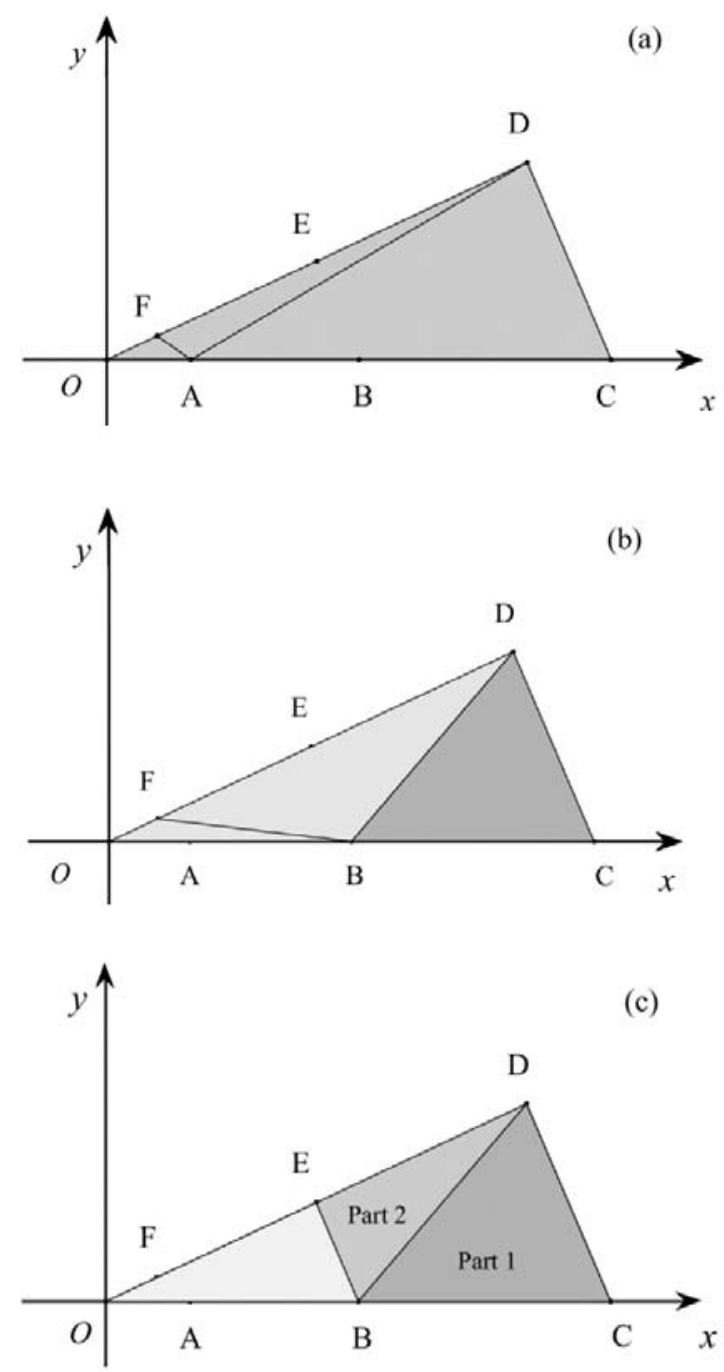

FIG. 2 Schematic diagram of the two-step mapping for one section (a) virtual space (b) intermediate space (c) physical space. 
In the second step, the points in BCD of FIG. 2c are the same as the points in BCD of FIG. 2b and thus

$$
\left\{\begin{array}{l}
x_{I I}=x_{I} \\
y_{I I}=y_{I}
\end{array}\right.
$$

The points in FBD of FIG. 2b are mapped to EBD of FIG. 2c by

$$
\left\{\begin{array}{c}
x_{I I}=c_{6} x_{I}+c_{7} y_{I}+c_{8} \\
y_{I I}=c_{9} x_{I}+c_{10} y_{I}+c_{11}
\end{array}\right.
$$

where,

$c_{6}=1+\frac{x_{D}\left(x_{F}-x_{E}\right)}{x_{B}\left(x_{D}-x_{F}\right)}$

$c_{7}=\frac{x_{D}\left(x_{D}-x_{B}\right)\left(x_{E}-x_{F}\right)}{x_{B} y_{D}\left(x_{D}-x_{F}\right)}$

$c_{8}=\frac{x_{D}\left(x_{E}-x_{F}\right)}{x_{D}-x_{F}}$

$c_{9}=\frac{y_{E}-y_{D}}{y_{F}-y_{D}} \frac{y_{F}}{x_{B}}-\frac{y_{E}}{x_{B}}$

$c_{10}=\frac{x_{B}\left(y_{E}-y_{D}\right)+x_{D}\left(y_{F}-y_{E}\right)}{x_{B}\left(y_{F}-y_{D}\right)}$

$c_{11}=\frac{y_{D}\left(y_{F}-y_{E}\right)}{y_{F}-y_{D}}$

After these two steps, then the points in ACD of FIG. 2a are mapped to the points in BCD of FIG. 2c using the relationship

$$
\left\{\begin{array}{l}
x_{I I}=c_{1} x+c_{2} y+c_{3} \\
y_{I I}=y
\end{array}\right.
$$

The points in FAD of FIG. 2a are finally mapped to points in EBD of FIG. 2c, using

$$
\left\{\begin{array}{l}
x_{I I}=c_{4} c_{6} x+\left(c_{5} c_{6}+c_{7}\right) y+c_{8} \\
y_{I I}=c_{4} c_{9} x+\left(c_{5} c_{9}+c_{10}\right) y+c_{11}
\end{array}\right.
$$

Next, the Jacobian matrix is defined as

$$
\boldsymbol{J}=\left[\begin{array}{ll}
\frac{\partial x_{I I}}{\partial x} & \frac{\partial x_{I I}}{\partial y} \\
\frac{\partial y_{I I}}{\partial x} & \frac{\partial y_{I I}}{\partial y}
\end{array}\right]
$$

From the transformation relations given in Equation (5), the Jacobian matrix for BCD is derived as

$$
\boldsymbol{J}_{1}=\left[\begin{array}{cc}
c_{1} & c_{2} \\
0 & 1
\end{array}\right] .
$$

Similarly, the Jacobian matrix for EBD (Equations. (6)) is derived as

$$
\boldsymbol{J}_{2}=\left[\begin{array}{ll}
c_{4} c_{6} & c_{5} c_{6}+c_{7} \\
c_{4} c_{9} & c_{5} c_{9}+c_{10}
\end{array}\right] .
$$

Then the density tensor and bulk modulus can be obtained, respectively, from the Jacobian matrix ${ }^{4}$ using

$$
\begin{gathered}
\overline{\boldsymbol{\rho}}=\rho_{0} \operatorname{det}(\boldsymbol{J})\left(\boldsymbol{J} \boldsymbol{J}^{T}\right)^{-1} \text {, and } \\
\boldsymbol{\kappa}=\boldsymbol{\kappa}_{0} \operatorname{det}(\boldsymbol{J}) .
\end{gathered}
$$

It is important to note that since $\boldsymbol{J}_{1}$ and $\boldsymbol{J}_{2}$ are independent of $x$ and $y$, indicating that the properties of each part are homogeneous.

The density tensors derived with Equation (10) are symmetric and can be transformed to a diagonal matrix. ${ }^{26}$ The eigenvalues of the density tensor, which represent the principal densities, will be denoted as $\rho_{m}$ and $\rho_{n}$. The speeds of sound along the two principal directions, $v_{m}$ and $v_{n}$, are different, which are calculated from the densities and bulk modulus in Equation. (11) for each principal direction using

$$
v=\sqrt{\kappa / \rho} .
$$

Having no off-diagonal elements in the density tensor, the cloak can now be approximated by layered structures, providing that the thickness of each layer is much smaller than the wavelength. ${ }^{36-37}$ The alternating layers form an anisotropic medium. With two alternately arranged, homogeneous, and isotropic layers A (mass density $\rho_{A}$, bulk modulus 
$\kappa_{A}$ ) and B (mass density $\rho_{B}$, bulk modulus $\kappa_{B}$ ), as shown in FIG. 3, the effective normal and in-plane densities and bulk moduli are computed using:

$$
\begin{gathered}
\rho_{\perp}=\frac{\rho_{A}+\eta \rho_{B}}{1+\eta}, \\
\frac{1}{\rho_{\|}}=\frac{1}{1+\eta}\left(\frac{1}{\rho_{A}}+\frac{\eta}{\rho_{B}}\right), \text { and } \\
\frac{1}{\kappa}=\frac{1}{1+\eta}\left(\frac{1}{\kappa_{A}}+\frac{\eta}{\kappa_{B}}\right),
\end{gathered}
$$

where $\eta=d_{A} / d_{B}$ is the thickness ratio of the alternating layers.

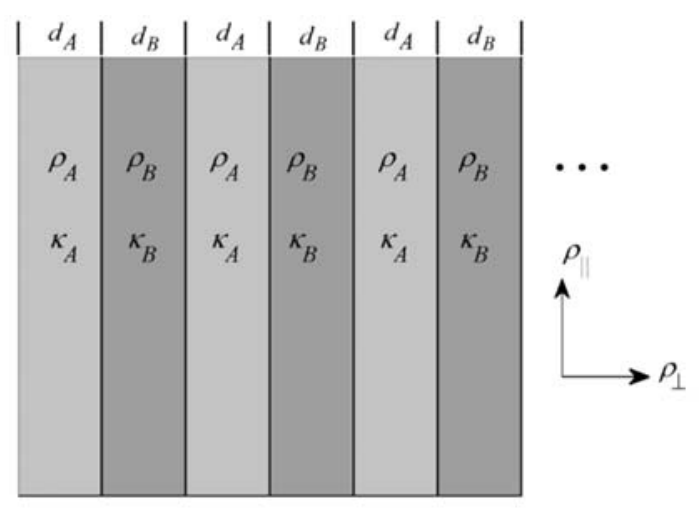

FIG. 3 Alternating layers of two homogeneous materials.

Letting $\rho_{m}>\rho_{n}$ and $\eta=1$, to get the effective properties for each part, the properties for layers $A$ and $\mathrm{B}$ are computed from

$$
\begin{gathered}
\rho_{A, B}=\rho_{m} \pm \sqrt{\rho_{m}^{2}-\rho_{m} \rho_{n}}, \text { and } \\
\kappa_{A, B}=\kappa .
\end{gathered}
$$

In each part of the cloak, $\rho_{m}, \rho_{n}$ are constant, resulting in constant values for $\rho_{A}, \kappa_{A}, \rho_{B}$, and $\kappa_{B}$. Having homogeneous properties in each part results in easier fabrication of the cloaking structures.

\section{Simulation}

The presented method can be used to design arbitrarily shaped cloaks. As a first example, a cloak is designed and simulated where the inner and outer boundaries of the cloak are both regular hexagons with a normalized inner side length of 1 and an outer side length of 2, as shown in FIG. 4.

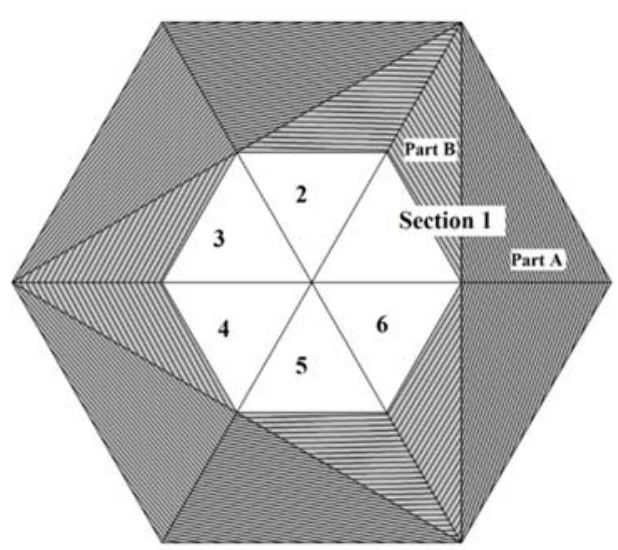

FIG. 4 Hexagonal cloak showing the layered structure.

The cloak is divided into six sections. The angle $\theta$ for each section is $\pi / 3$. Letting the lengths $|\mathrm{OA}|$ and $|\mathrm{OF}|$ (FIG. 2) be 0.1, then the properties of the sections can be derived using equations (1)-(15). After the two-step transformation, each of the six sections are divided into two triangular parts and built with layered structures along principal directions. It should be noted that the division of the hexagonal cloak is not unique. It is possible to divide it into smaller sections. The division affects the properties of each part, meaning that the number of sections, and thus $\theta$, can be also used to tailor the properties of the cloak.

Full wave simulations for the total pressure field around the inner hexagonal obstruction with and without the hexagonal cloak were conducted with COMSOL Multiphysics finite element analysis software. The amplitude of background pressure waves is $1 \mathrm{~Pa}$. The results are shown in FIG. 5. It can be seen that the cloak reduces the scattering and shadows significantly. 

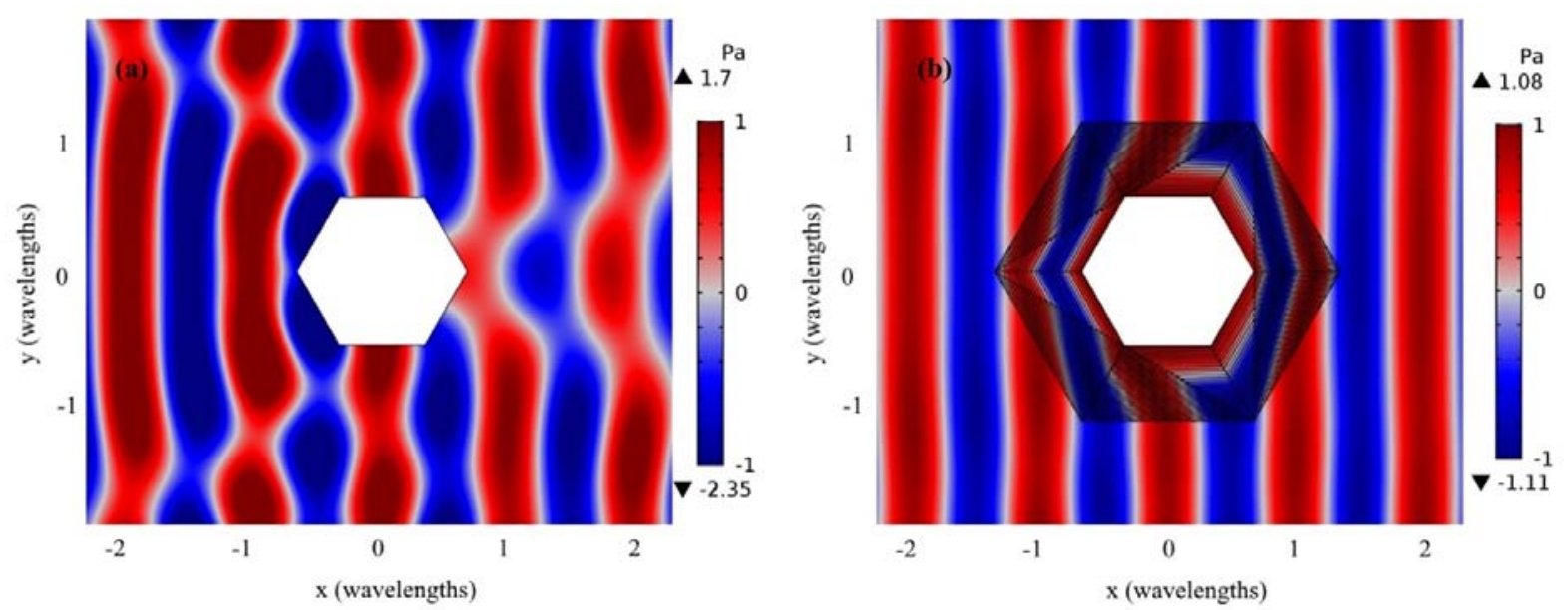

FIG. 5 The contour plot of the acoustic pressure fields for plane waves (1 Pa) (a) without and (b) with the hexagonal cloak.

Cloaks with curvilinear boundaries can also be approximated with piecewise linear segments. To illustrate, a more general cloak is designed and simulated next. The shape of the cloak is irregular. There are 32 sections after division and the angles of the sections are not the same (more divisions in regions of higher curvature). The properties of each of these sections are derived respectively. $|\mathrm{OA} / / \mathrm{OB}|$

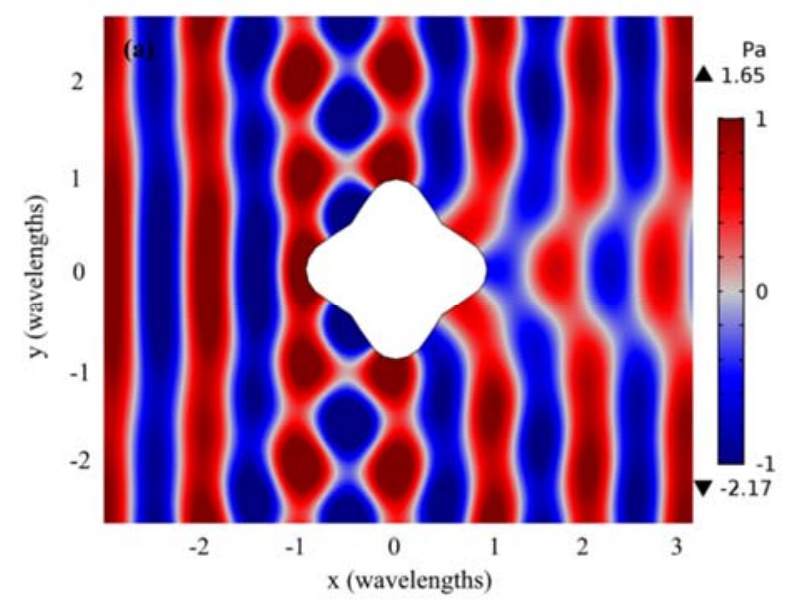

and $|\mathrm{OF} / /| \mathrm{OE} \mid$ are both 0.1 for every section.

Full wave simulations for the total pressure field with and without the irregular cloak were conducted with COMSOL Multiphysics finite element analysis software, with results shown in FIG. 6. The amplitude of background pressure waves is $1 \mathrm{~Pa}$. It can be seen from the figure that the cloak works well at reducing scattering and shadows.

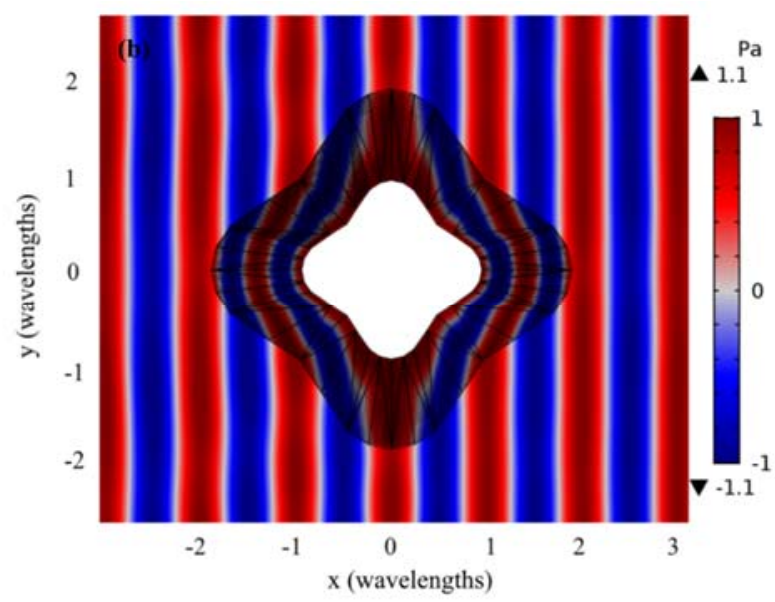

FIG. 6 The contour plot of the acoustic pressure fields for plane waves (1 Pa) (a) without and (b) with the irregular cloak.

The properties of cloaks are usually continuously variable and not physically realizable. Not only does performing the two-step transformation create cloaks with homogeneous parts, but the parameters can be adjusted to further make the properties more amenable to being realized.
For a regular polygonal cloak which is divided into the number of its sides (let $|\mathrm{OB}|=|\mathrm{OE}|=1$, and $|\mathrm{OC}|=|\mathrm{OD}|=2)$, there are three parameters that determine the properties of the cloak: the normalized lengths $|\mathrm{OA}|$ and $|\mathrm{OF}|$, and the angle $\theta$. The effects of varying the geometries $(\theta,|\mathrm{OA}|$, and the ratio of 
$|\mathrm{OF}| /|\mathrm{OA}|)$ on the relative principal velocities, $v_{i j}$ (first subscript is which part and second is direction), are given in FIGs. 7-9. When $\theta$ is constant and $|\mathrm{OA}|=|\mathrm{OF}|$, the principal velocities (Equation 12) deviate from that of the background material as $|\mathrm{OA}|$ is decreased, as shown in FIG. 7. Strong dependence on $|\mathrm{OA}|$ is seen in $v_{2 \mathrm{n}}$ in particular. Similarly, when $|\mathrm{OA}|$ and $|\mathrm{OF}|$ are constant and equal, the relative principal velocities can also be varied from that of the background material by changing $\theta$, as shown in FIG. 8. Finally, no variation of principal velocities for the first part was found, while the variation for second part with the ratio of $|\mathrm{OF}| /|\mathrm{OA}|$ is shown in FIG. 9 for various angles. For each angle, there is a value leading to the minimum difference of the velocities with respect to that of the background.

In order to guarantee good cloaking performance, the values of $|\mathrm{OA}|$ and $|\mathrm{OF}|$ should be as small as possible. But extremely small values correspond to extreme material properties which are difficult to realize. The three geometric parameters can be tailored to strike a balance between good cloaking performance and material properties that are amenable to fabrication. Additional simulations were run which varied $|\mathrm{OA}|=|\mathrm{OF}|$ over a range of values and found that a value of 0.2 provides good cloaking performance and material properties that are within the natural range (For $|\mathrm{OA}|=0.2,|\mathrm{OF}|=0.2, \theta=\pi / 9$ $\left(20^{\circ}\right)$, the relative principal velocities are $v_{1 \mathrm{n}}=1.00$, $v_{1 \mathrm{~m}}=0.553, v_{2 \mathrm{n}}=5.06, v_{2 m}=0.549$ ).

With previous one-step transformations, the properties of the cloaks are inhomogeneous and the properties near the inner boundary are extremely large or small. ${ }^{26}$ With the two-step transformation, the cloaks are composed of homogeneous parts, the properties of which can be adjusted by varying the geometric parameters, making it easier to fabricate the cloaks.

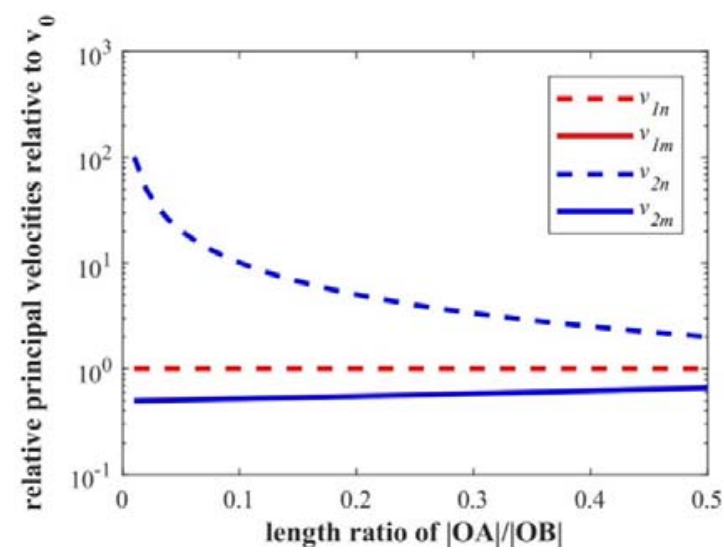

FIG. 7 Variation of relative principal velocities with $|\mathrm{OA}|$ under the conditions of $\theta=\pi / 10$ and $|\mathrm{OF}| /|\mathrm{OA}|=1$.

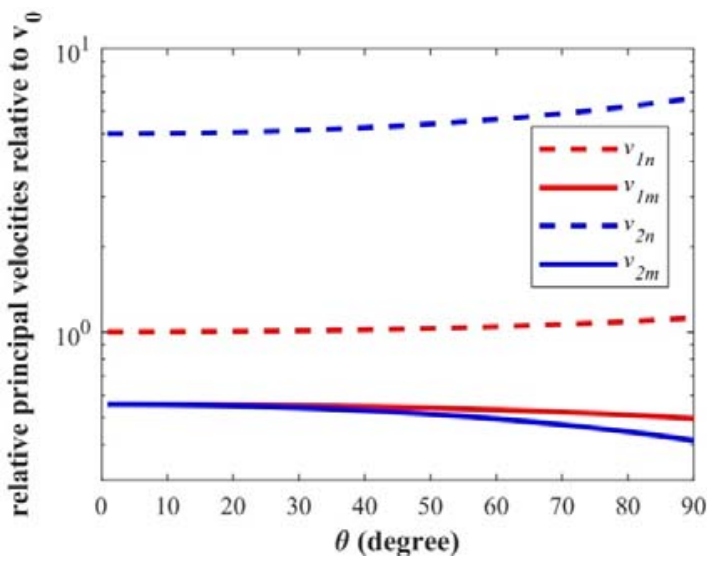

FIG. 8 Variation of relative principal velocities with $\theta$ under the conditions of $|\mathrm{OA}|=|\mathrm{OF}|=0.2$.

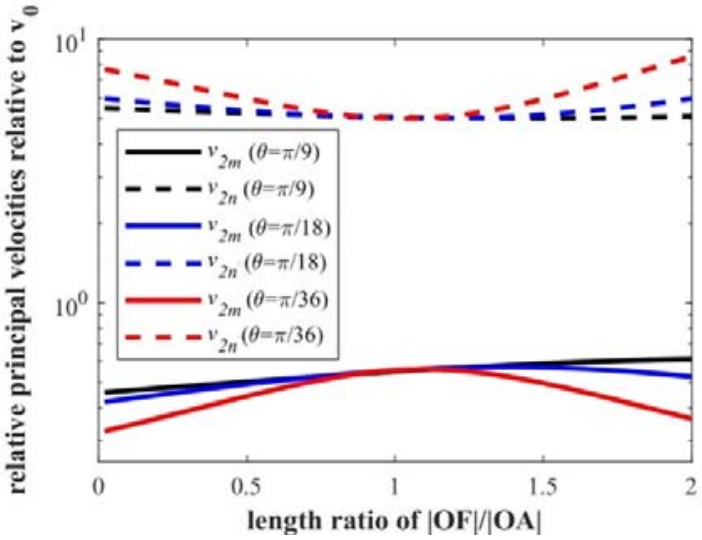

FIG. 9 Variation of relative principal velocities with $|\mathrm{OF}| /|\mathrm{OA}|$ under the condition of $|\mathrm{OA}|=0.2$ for various values of $\theta$. 


\section{Conclusion}

A two-step transformation method is proposed for the design of acoustic cloaks with arbitrary shapes. Although the properties are anisotropic, they can be approximated with layered structures. The density and bulk modulus of traditionally designed annular cloaks are inhomogeneous, since they are functions of radius. What's more, the material properties of the cloak become extreme near the inner boundary. In contrast, the design method used in this paper produces cloaks that are composed of homogeneous parts, which can reduce difficulty when fabricating the cloaks.

The parameters $|\mathrm{OA}|$ and $|\mathrm{OF}|$ and the angle $\theta$ used in the transformations affect the required properties of the cloaks as well as the performance. Through proper selection of $|\mathrm{OA}|,|\mathrm{OF}|$, and $\theta$, a balance can be made between cloaking performance and cloak properties, producing cloaks that are physically realizable.

Full wave simulations were conducted for two models: a hexagonal cloak and an irregular, curved cloak. The results show that the cloaks work well at reducing the reflections and shadows.

\section{Reference}

${ }^{1}$ J. B. Pendry, D. Schurig, and D. R. Smith, Science, 312, 1780 (2006).

${ }^{2}$ S. A. Cummer, and D. Schurig, New Journal of Physics, 9, 45 (2007).

${ }^{3}$ S. A. Cummer, B. I. Popa, D. Schurig, D. R. Smith, J. Pendry, M. Rahm, and A. Starr, Physical review letters, 100, 024301. (2008).

${ }^{4} \mathrm{H}$. Chen, and C. T. Chan, Applied physics letters, 91, 183518 (2007).

${ }^{5}$ H. Chen, Z. Liang, P. Yao, X. Jiang, H. Ma, and C. T. Chan, Physical review B, 76, 241104 (2007).

${ }^{6} \mathrm{H}$. Chen, T. Yang, X. Luo, and H. Ma, Chinese Physics Letters, 25, 3696 (2008).
${ }^{7} \mathrm{~J}$. Li, and J. B. Pendry, Physical review letters, 101, 203901 (2008).

${ }^{8}$ B. I. Popa, L. Zigoneanu, and S. A. Cummer, Physical review letters, 106, 253901 (2011).

${ }^{9}$ L. Zigoneanu, B. I. Popa, and S. A. Cummer, Nature materials, 13, 352 (2014).

${ }^{10}$ S. Zhang, C. Xia, and N. Fang, Physical Review Letters, 106, 024301 (2011).

${ }^{11}$ Y. Lai, H. Chen, Z. Q. Zhang, and C. T. Chan, Physical review letters, 102, 093901 (2009)

${ }^{12}$ X. Zhu, B. Liang, W. Kan, X. Zou, and J. Cheng, Physical review letters, 106, 014301 (2011).

${ }^{13}$ T. Xu, X. F. Zhu, B. Liang, Y. Li, X. Y. Zou, and J. C. Cheng, Applied Physics Letters, 101, 033509 (2012).

${ }^{14}$ W. Kan, B. Liang, X. Zhu, R. Li, X. Zou, H. Wu, J. Yang, and J. Cheng, Scientific reports, 3, 1427 (2013).

${ }^{15}$ A. N. Norris, Proceedings of the Royal Society of London A: Mathematical, Physical and Engineering Sciences, 464, 2411 (2008).

${ }^{16}$ C. L. Scandrett, J. E. Boisvert, and T. R. Howarth, Wave Motion, 48, 505 (2011).

${ }^{17}$ N. H. Gokhale, J. L. Cipolla, and A. N. Norris, The Journal of the Acoustical Society of America, 132, 2932 (2012).

${ }^{18}$ Y. Chen, X. Liu, and G. Hu, Scientific reports, 5, 15745 (2015).

${ }^{19}$ X. Zhu, H. Ramezani, C. Shi, J. Zhu, and X. Zhang, Physical Review X, 4, 031042 (2014)..

${ }^{20}$ Y. G. Peng, C. Z. Qin, D. G. Zhao, Y. X. Shen, X. Y. Xu, M. Bao, H. Jia, and X. F. Zhu, Nature communications, 7, 13368 (2016).

${ }^{21}$ Q. Wu, K. Zhang, F. Y. Meng, and L. W. Li, Journal of Physics D: Applied Physics, 42, 035408 (2008). 
22J. Zhang, Y. Luo, H. Chen, and B. I. Wu, JOSA B, 25, 1776 (2008).

${ }^{23}$ W. X. Jiang, J. Y. Chin, Z. Li, Q. Cheng, R. Liu, and T. J. Cui, Physical Review E, 77, 066607 (2008).

${ }^{24}$ H. Ma, S. Qu, Z. Xu, and J. Wang, Optics express, 16, 15449 (2008).

${ }^{25}$ O. Paul, Y. Urzhumov, C. Elsen, D. Smith, and M. Rahm, Journal of Applied Physics, 111, 123106 (2012).

${ }^{26} \mathrm{Q}$. Li, and J. S. Vipperman, Applied Physics Letters, 105, 101906 (2014).

${ }^{27}$ B. I. Popa, and S. A. Cummer, Physical Review A, 82, 033837 (2010).

${ }^{28}$ B. I. Popa, and S. A. Cummer, Journal of Optics, 18, 044018 (2016).

${ }^{29}$ Y. Luo, J. Zhang, H. Chen, S. Xi, and B. I. Wu, Applied Physics Letters, 93, 033504 (2008).
${ }^{30}$ A. Rajput, and K. V. Srivastava, Journal of Applied Physics, 116, 124501 (2014).

${ }^{31}$ S. A. Cummer, R. Liu, T. J. Cui, Journal of Applied Physics 105, 056102 (2009).

${ }^{32}$ W. Li, J. Guan, Z. Sun, W. Wang, and Q. Zhang, Optics express, 17, 23410 (2009).

${ }^{33}$ X. Wang, S. Qu, X. Wu, J. Wang, Z. Xu, and H. Ma, Journal of Physics D: Applied Physics, 43, 305501 (2010).

${ }^{34} \mathrm{~T}$. Li, M. Huang, J. Yang, Y. Lan, and J. Sun, Journal of Vibration and Acoustics, 134, 051016 (2012).

${ }^{35}$ R. Zhu, B. Zheng, C. Ma, J. Xu, N. Fang, and H. Chen, The Journal of the Acoustical Society of America, 140, 95 (2016).

${ }^{36}$ Y. Cheng, F. Yang, J. Y. Xu, and X. J. Liu, Applied Physics Letters, 92, 151913 (2008).

${ }^{37} \mathrm{D}$. Torrent, J. Sánchez-Dehesa, New Journal of Physics, 10, 063015 (2008). 